\title{
AFM CHARACTERIZATION OF NANOCOMPOSITE SAMPLES
}

\author{
1'Anna CHARVÁTOVÁ CAMPBELL, 1,2Marek HAVLIČEK, 1,2Petr KLAPETEK, ${ }^{3}$ Vilma BURŠíKOVÁ \\ ${ }^{1}$ Czech Metrology Institute, Brno, Czech Republic, EU, acharvatovacampbell@cmi.cz \\ ${ }^{2}$ Central European Institute of Technology, Brno, Czech Republic, EU \\ ${ }^{3}$ Faculty of Science, Masaryk University, Brno, Czech Republic, EU
}

https://doi.org/10.37904/nanocon.2020.3775

\begin{abstract}
Nanocomposite polymer coatings combine attractive functionalities from the combination of organic matrix coatings and inorganic nanoparticles which allows to acquire unique properties. They can be tailored for specific needs by modifying optical, mechanical, electronic, magnetic and other properties, which may differ significantly from the properties of the bulk form.

In order to study the role of the incorporated nanoparticles, topography is of fundamental importance. Its relation to the deposition parameters, however, is far from trivial. Suitable statistical parameters, which capture the essential traits of the sample but are not oversensitive to local variations are needed. Roughness e.g. is a well-established, popular statistical parameter but is not sufficient to describe the topography. Parameters which describe lateral distances should be included as well. In order to ensure the same processing for multiple samples the data processing should also require as little user input as possible. As a wide range of samples must be characterized, time demands for the characterization of a single sample should also be considered.

We present a systematic atomic force microscopy study of a series of nanocomposite polymer coatings with topographies including spherical particles and snake-like structures of different length scales. Different statistical parameters, their dependence on measurement settings and related uncertainties are addressed.
\end{abstract}

Keywords: AFM, nanocomposites

\section{INTRODUCTION}

Nanocomposite polymer coatings combine attractive functionalities from the combination of organic matrix coatings and inorganic nanoparticles which allows to acquire unique properties. They can be tailored for specific needs by modifying optical, mechanical, electronic, magnetic and other properties, which may differ significantly from the properties of the bulk form.

Deposition under dusty plasma condition can be used for depositing nanocomposite thin films, with many successful technological applications. Varying the deposition conditions allows to obtain films with different properties, such as elasticity or hydrophobicity [1-6].

The topography is one of the key features of a film which must be characterized. Atomic force microscopy (AFM) is very well suited for this task. However, it is necessary to keep in mind several issues. Firstly, AFM measurements can be time demanding, especially if large numbers of samples should be characterized. It is thus necessary to carefully choose parameters for the measurement such as resolution, image size, scanning rate, etc. as these may affect the validity of the data. Secondly, AFM measurements need further data processing, such as levelling, row alignment, removal of scars or other artifacts. These procedures can significantly affect the results [7]. Therefore, procedures should be exactly defined with as little user choices as possible in order to ensure that all samples will be processed in the same way. Automated procedures can help to achieve this as well as reduce the time necessary to accomplish this. 
A large number of statistical parameters and functions can be evaluated, for an overview see e.g. [8]. The most popular ones include the root mean square, skew, kurtosis, surface area, power spectral density function, autocorrelation function, and Euler characteristics. Further analysis is possible by marking grains and evaluating their size parameters. However, for the comparison of different samples the focus should be on reproducibility more than on the theoretical interpretation of the chosen parameter.

In this work we present the analysis of AFM measurements on a series of samples obtained by PECVD depositions in dusty plasma and suggest optimal parameters for their statistical description, with focus on their robustness.

\section{METHODS}

In this work we analyzed a set of 65 samples. The samples were produced using plasma enhanced chemical vapor deposition from hexamethyldisiloxane (HMDSO) and oxygen. The details of the deposition process are described in [6]. A capacitive radio frequency discharge at $13.56 \mathrm{MHz}$ was generated in a parallel plate reactor. The HMDSO and oxygen flow rates varied from 1 to $10 \mathrm{sccm}$ and 2 to $10 \mathrm{sccm}$, respectively. Single crystal silicon wafers were used as substrates. The applied power ranged from 25 to $75 \mathrm{~W}$ and the DC negative selfbias voltage between -10 to $-400 \mathrm{~V}$. The deposition conditions led to the creation of dusty plasma.

AFM measurements were performed using a Dimension Icon instrument from Bruker. Measurements were performed in tapping and Bruker's PeakForce tapping mode. Images of different sizes were measured ranging from $2 \mu \mathrm{m} \times 2 \mu \mathrm{m}$ to $40 \mu \mathrm{m} \times 40 \mu \mathrm{m}$ with resolution usually $512 \times 512$ or $1024 \times 1024$.

Data were leveled by subtracting a fitted polynomial of second degree. Lines were aligned with respect to the line median. Standard statistics were extracted, such as average, rms, skew, kurtosis, surface area, as well as the radial power spectral density function and autocorrelation. All data processing was performed within the open source software Gwyddion (www.gwyddion.net).

\section{RESULTS}

Several types of topography have been encountered in this study: the most common were different types of spherical nanoparticles, either individually placed on a flat surface or forming large clusters, snake-like structures and caterpillar-like structures formed by spherical particles aligning themselves into snakes. The vertical size of objects ranged between $30 \mathrm{~nm}$ and $730 \mathrm{~nm}$. The lateral size of the objects ranged from $200 \mathrm{~nm}$ to $2 \mu \mathrm{m}$. The thickness varied between $500 \mathrm{~nm}$ and $3 \mu \mathrm{m}$.

The root mean square roughness and the ratio of surface area and projected area have been found to be particularly useful and are shown in Figure 1, together with some examples of topographies. As can be seen, the root mean square value of an image is only very weakly correlated with the type of the surface. The ratio of surface and projected area is more significant, notably large values have been found to correspond always to snake-like structures. A similar study of skew and excess kurtosis didn't show any significant trends, though particles tended to have positive skew whereas snakes and caterpillars had both positive and negative skews. Especially for larger structures we should check the effect of the image size on the evaluated value. Some recommendations are given in standards [9-11] but their use in this case is unclear. Instead, we performed studies on selected samples which were measured with larger scan size.

From a $40 \mu \mathrm{m} \times 40 \mu \mathrm{m}$ image details of different size were extracted and processed. The variance of the different statistical parameters was estimated with respect to the location of the detail using a Monte Carlo method. In Figure 2 we show the dependence of the roughness and excess kurtosis on the size of the detail, and the dependence of the relative uncertainty of the parameters on the size. From Figure 2B we can see that for this sample the roughness could be estimated with an uncertainty around $1 \mathrm{~nm}$ already at $10 \mu \mathrm{m}$. This corresponds to an uncertainty of the excess kurtosis of 0.2 which is acceptable. From Figure $2 \mathbf{C}$ it is obvious 
that the sensitivity of the parameter grows with growing order of the moment involved. Results for other samples are similar.
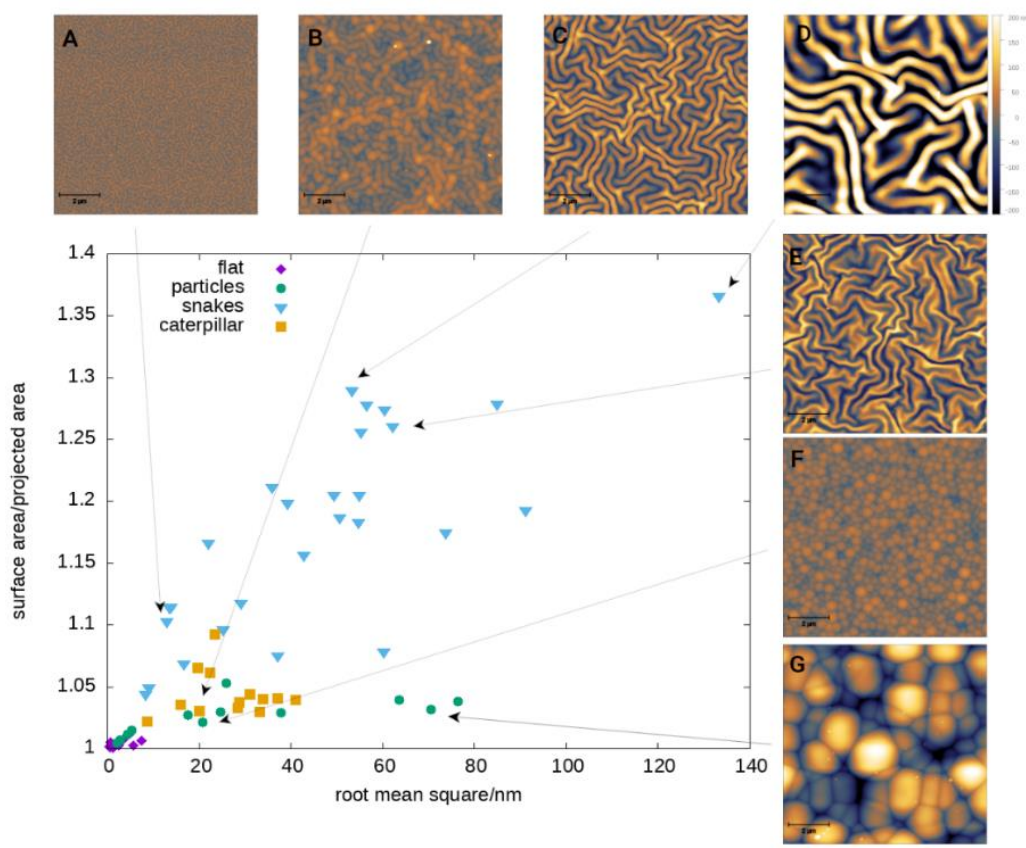

Figure 1 The root mean square value and the ratio of the surface area and projected area for the samples involved in this study. Some typical examples of topographies encountered are shown. The vertical ranges have been chosen the same for all images for better comparison.
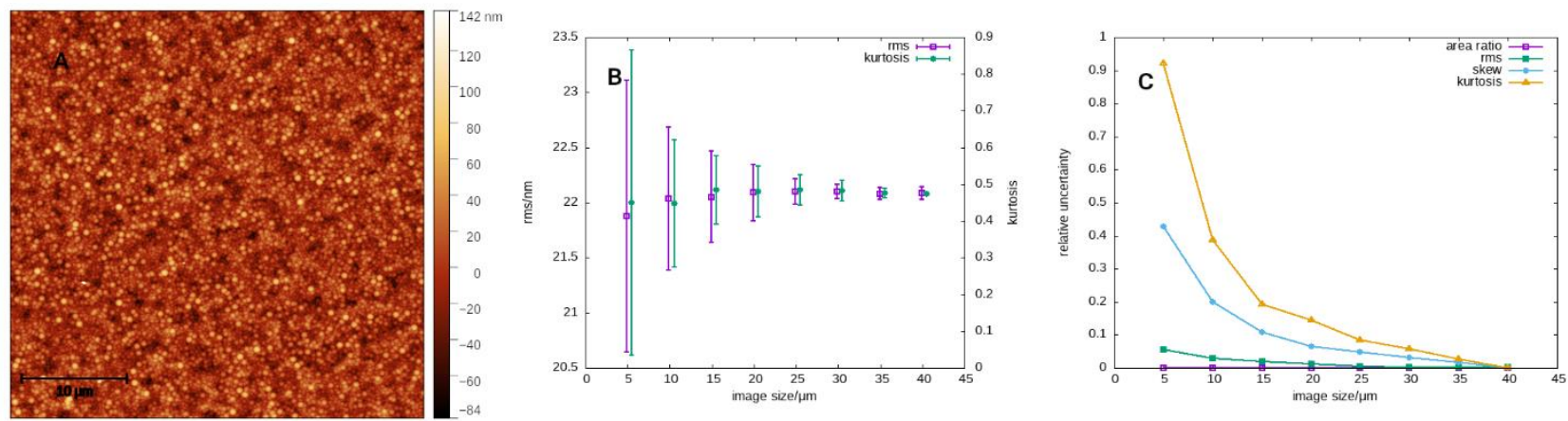

Figure 2 AFM image used for studying the dependence of statistical parameters on scan size (A), the dependence of the roughness and excess kurtosis on the scan size $(B)$ and the dependence of relative uncertainty of statistical parameters on the size $(C)$. Data have been shifted in the image $B$ for better clarity.

As shown in [12], the root mean square roughness as well as skew and kurtosis are very sensitive to levelling and scan line corrections. On the other side, the surface area is largely unaffected. We found that the samples in this study had to be levelled using polynomials of at least second degree. The recommendations of the authors hold for random rough surfaces, but using them we find that the scan length should be approx. 20 times longer than the characteristic length scale in order to keep the bias from the true value below roughly $10 \%$. This would correspond to image sizes ranging between $4-40 \mu \mathrm{m}$. This is in reasonable agreement with the requirements based on the (in)homogeneity.

One common method for the analysis of AFM images of nanoparticles is to mark them using some of the wellestablished algorithms, see e.g. $[13,14]$ and then perform a statistical analysis of these. However, in most 
cases this requires the choice of certain thresholds which must be made by the user. The resulting statistics may be very sensitive to this choice and there is no simple way to define the thresholds universally for all samples. Therefore, the results are not comparable between samples. Last but not least, this method is time consuming and cannot be easily automated.

However, some insight can be gained by using the mean curvature. The mean curvature is given as the average of the principal curvatures. For concave ellipsoids both principal curvatures are positive, for concave cylinders one is positive and the other zero. Thus, we expect a positive mean curvature to correspond mainly to concave areas, which can be either ellipsoidal (nanoparticles) or cylindrical (snakes), and assume other cases are less common and can be omitted. Since the mean curvature is calculated based on numerical derivatives, it is very sensitive to noise and some kind of smoothing may be necessary. We also encountered voids in the marked grains which require further processing for a rigorous, automated statistical evaluation.

Surfaces formed by nanostructures such as particles or snakes should be characterized also from the viewpoint of lateral length scales. Notably, the radius of the particles and snakes is of crucial interest. Obviously, this could be measured manually directly from the image. This is a lengthy procedure and can be biased by the user's choice.

For rough surfaces models one of the common parameters to characterize its lateral scale is the correlation length where the autocorrelation function (ACF) decays to 1/e of its maximum [8]. This corresponds to Gaussian and exponential models of roughness and does not have a simple interpretation for geometries encountered in this study. Nevertheless, the correlation length can be easily determined unambiguously. Alternatively, the Fourier transform of the autocorrelation function, the power spectral density function (PSDF), can also be used for the identification of characteristic length scales. The fitting procedures using PSDF may be favorable for numerical reasons.

For individual particles models of the ACF have been found in [15] from which the shape parameters can then be extracted. However, this model assumes a fairly low coverage and notably the existence of a reference plane on which the particles are placed. In our case the shapes found in the samples are too irregular for this model but we can still use the peaks of the ACF and PSDF as characteristic length scales. As there is a big variability in the angular directions, we use the angular average for both for ACF and PSDF, the radial autocorrelation function (RACF) and radial power spectral density function (RPSDF) in all cases.
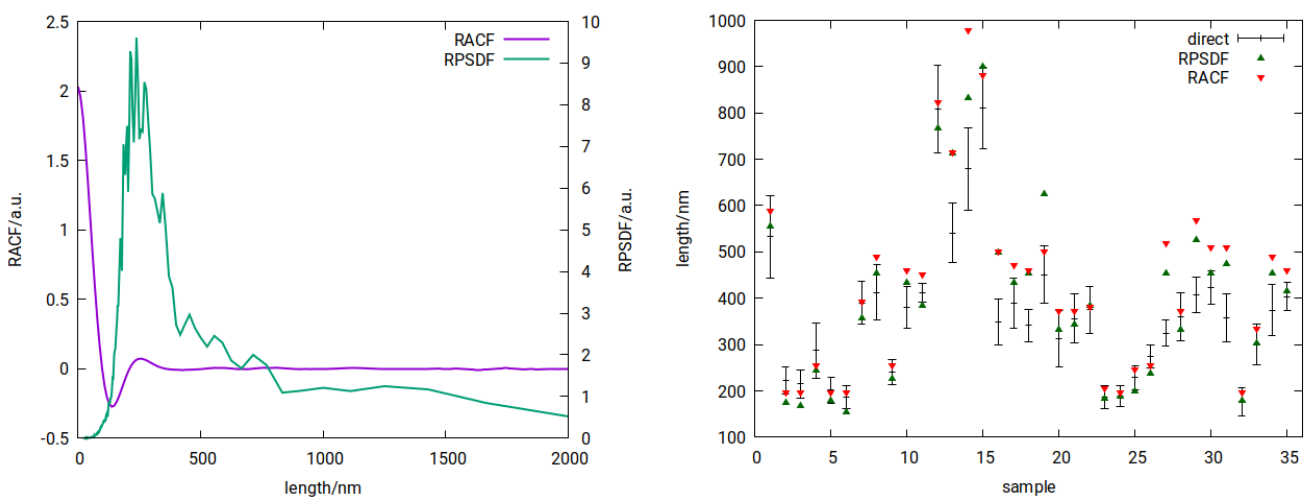

Figure 3 Example of typical RACF and RPSDF (left), characteristic length scale of particles for different samples obtained by identification of peaks in RPSDF, identification of peaks in RACF and by direct measurement from image

An example of the RACF and RPSDF and the corresponding length scales are shown in Figure 3. The correspondence with the direct measurement is reasonable, mainly due to the large uncertainty of the latter. This reflects that the image size was too small. The difference between RACF and RPSDF is on the order of tens of nanometers, which is acceptable for a pixel size of $9 \mathrm{~nm}$. The methods failed on samples formed by 
particles or snakes placed on top of each other forming hills and valleys, such as e.g. image G in Figure 1. The RACF method failed more often than the RPSDF method (14 and 8 out of 51 cases).

In contrast the length scale based on the decay of the RACF is simple to obtain and can be easily automated. However, this value does not offer any direct physical interpretation. No trend for the ratio of the decay rate and the length scale measured directly has been found. For the data shown in Figure 4 the ratio varies between 0.2 and 0.65 .

We illustrate the algorithms described so far for a study of the effect of deposition time with other deposition conditions kept constant. The AFM images of such samples are shown in Figure $\mathbf{4}$ where the mean curvature is positive.

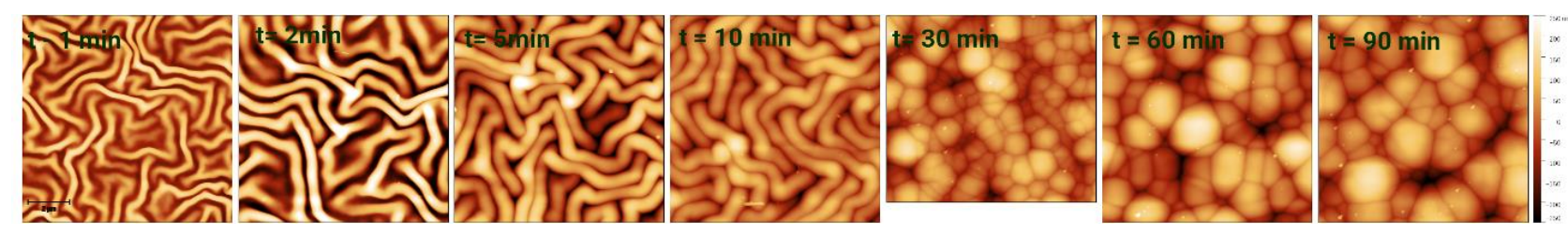

Figure 4 AFM images of a series of samples corresponding to different deposition times with otherwise identical deposition conditions

The dependence of the roughness, area ratio, number of grains marked by the mean curvature, the correlation length defined by the decay of the RACF and the size measured directly from the image on the deposition time is shown in Figure 5. The length scale could not be determined from the RACF and RPSDF, due to the nonplanarity of the surface, as explained above. The samples had been measured at the fixed scan size of $10 \mu \mathrm{m}$. This is not sufficient for samples with particles as large as the ones in this series but offers a good first estimate.
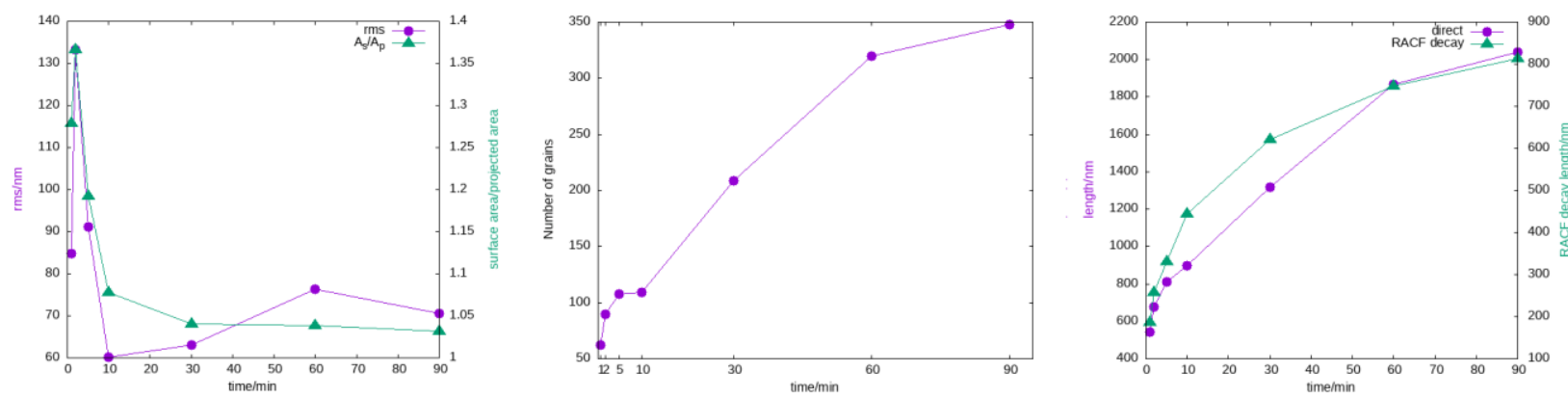

Figure 5 The time dependence of roughness and surface ratio (left), of the number of grains (middle), and of lateral scale (right). The particle size determined directly from image and RACF decay rate were used.

Care is needed for the AFM measurement itself. The scan size must be sufficiently large so as to capture inhomogeneities and offer sufficient quality of RACF and RPSDF. Artifacts such as line mismatch or contamination can significantly affect the analysis and should be corrected for. However, the correction process must be done manually which increases time demands and may introduce unintended bias due to the user choices. Large images are more prone to artifacts as the scan rate cannot be kept as low as for small images, thermal drift being one of the major issues. As a first estimate, the image size should be at least 20 times larger than characteristic length, which can be estimated as the diameter of particles or snakes

\section{CONCLUSION}

As it was found that the deposition process leads to a range of very different surface topographies, our goal was to find suitable statistical parameters for describing the surface topography in such a wide set. We have 
shown that a high ratio of surface area and projected area is related to snake like structures. Masks based on the mean curvature were shown to be capable of grain identification. Different methods of identifying length scales were compared to direct measurements. The determination of length scales from peaks in RPSDF and RACF is preferable since it produces physically meaningful results.

The usefulness of these parameters has been manifested on a series of samples which were prepared under identical deposition conditions with only the deposition time varying. The interpretation of the results from the perspective of the deposition process shall be presented elsewhere.

\section{ACKNOWLEDGEMENTS}

\section{This work was supported by the Czech Science Foundation project GACR 19-15240S "Multifunctional Nanocomposite Polymer Thin Films with Controlled Surface and Mechanical Properties Deposited in RF Dusty Plasma.}

\section{REFERENCES}

[1] FACIO, D. S. MOSQUERA, M. J. Simple Strategy for Producing Superhydrophobic Nanocomposite Coatings in situ on a Building Substrate. ACS Appl. Mater. Interfaces. 2013, vol. 5, pp. 7517-7526.

[2] BOUFENDI, L. BOUCHOULE, A. Particle nucleation and growth in a low-pressure argon-silane discharge. Plasma Sources Sci. Technol. 1994, vol. 3, pp. 262.

[3] CAVARROC, M. et al. Self-excited instability ocurring during the nanoparticle formation in an $\mathrm{Ar}$-SiH4 low pressure radio frequency plasma. J. App. Phys. 2006, vol. 99, pp. 064301.

[4] CABARROCAS, P. R. et al. Synthesis of silicon nanocrystals in silane plasmas for nanoelectronics and large area electronic devices. J. Phys. D: Appl. Phys. 2007, vol. 40, pp. 2258.

[5] BEHLER, K. et al. Nanodiamond-Polymer Composite Fibers and Coatings. ACS Nano. 2009, vol. 3, pp. 363-369.

[6] CHARVÁTOVÁ CAMPBELL, A., KLAPETEK, P., VALTR, M., BURŠíKOVÁ, V. Development of reference materials for the investigation of local mechanical properties at the nanoscale. Surf. Interface Anal. 2012, vol. 44, pp. 1151.

[7] KLAPETEK, P. NEČAS, D. Study of user influence in routine SPM data processing. Meas. Sci. Technol. 2017, vol 28, pp. 034014-034024

[8] ANDERSON, T. W. The Statistical Analysis of Time Series. New York: Wiley, 1971.

[9] ASME B46.1. Surface texture (surface roughness, waviness, lay). Am. Soc. Mech. Eng. 2009.

[10] ISO 19606. Fine ceramics (advanced ceramics, advanced technical ceramics) - Test method for surface roughness of fine ceramic films by atomic force microscopy. 2017.

[11] ISO 4288. Geometrical product specifications (GPS)—surface texture: Profile method-rules and procedures for the assessment of surface texture. 1996.

[12] NEČAS, D. et al. How levelling and scan line corrections ruin roughness measurement and how to prevent it. Sci.Rep. 2020, vol. 10, pp. 15294

[13] VINCENT, L. SOUILLE, P. Watersheds in digital spaces: an efficient algorithm based on immersion simulations. IEEE Trans. Pattern Anal. Mach. Intell. 1991, vol. 13, pp.586-598.

[14] OTSU, N. A Threshold Selection Method from Gray-Level Histograms. IEEE Trans. Syst. Man Cybern. Syst. 1979, vol. 9, pp. 62-66.

[15] MAREK, J. DEMJEN, E. Is autocorrelation image analysis the proper method in nanoparticle sizing? J. Nanopart. Res. 2017, vol. 19, pp. 208. 